\title{
AUGMENTATION OF ATROPHIC POSTERIOR MANDIBLE USING INLAY XENOGRAFT VERSUS AUTOGRAFT BONE BLOCKS
}

\author{
Mona Samy Sheta* , Mohamed Monay Shoushan**, Mohammad Abdelhakim Elshall**, \\ Ibrahim Mohamed Nowair*** and Eman Mohy El-Din Megahed ${ }^{* * * *}$
}

\begin{abstract}
Purpose: This study aimed to compare the use of interpositional bovine bone block to autogenous chin bone block in augmentation of atrophic posterior mandible for dental implant installation.
\end{abstract}

Materials and Methods: Twelve patients $(n=12)$ with atrophic posterior mandible were augmented using inlay technique and divided randomly into two equal groups according to the type of the graft used, autogenous bone graft (Group I) and bovine bone graft (group II). After six months, core biopsies sites were taken from the sites of implant, which were analyzed histologically.

Results: Six months after augmentation, alveolar bone height gain were $10.76 \pm 1.043 \mathrm{~mm}$ and $11.24 \pm 0.3721 \mathrm{~mm}$ for group I and group II respectively. There was no statistical significant difference in height gain between both groups as $p>0.05$. There was more graft remnants in bovine group, but both groups showed a good level of osteointegration with an adequate extension of bonding surface between host bone and the graft particles.

Conclusion: Both grafts achieved successful results, however the use of the bovine bone graft was less invasive and avoided donor site morbidity.

KEYWORDS: Interpositional grafting, atrophic posterior mandible, autogenous bone graft, bovine xenograft.

\section{INTRODUCTION}

The alveolar ridge undergoes a continuous resorptive process after teeth $\operatorname{loss}^{1}$. Excessive resorption of the alveolar ridge in a vertical direction may compromise possibility of implant placement. This will eventually result in insufficient bone superior to the inferior alveolar nerve (IAN), making dental implant placement impossible without performing augmentation of the alveolar bone in terms of height ${ }^{2}$.

\footnotetext{
* Associate Lecturer of Oral and Maxillofacial Surgery, Faculty of Dentistry, Tanta University, Egypt

** Professor of Oral and Maxillofacial Surgery, Faculty of Dentistry, Tanta University, Egypt

*** Associate Professor of Oral and Maxillofacial Surgery, Faculty of Dentistry, Tanta University, Horus University, Egypt **** Associate Professor of Oral pathology, Faculty of Dentistry, Tanta University, Egypt.
} 
Rehabilitation of the atrophic posterior mandible is a challenge in dental practice. Conventional treatments include the segmental sandwich osteotomy or inlay bone grafting (IBG), onlay bone grafting (OBG), short implants, distraction osteogenesis, and inferior alveolar nerve transposition (IANT) ${ }^{3}$.

The cornerstone in the bone regeneration procedures is the use of bone substitutes to create a bone mantle that covers the screw to enhance implant stability and treatment outcome. Bone graft materials can be divided in four large groups: Autografts, Allografts, Xenografts and Synthetic biomaterials, which provide a structural framework for clot development, maturation and remodeling that supports bone formation in osseous defects ${ }^{4}$.

Traditional distraction osteogenesis aims to maintain the majority of the vascularity to the transported bone segment. The drawbacks of distraction osteogenesis include patient cooperation, technique sensitivity, and the possibility of a second surgery to remove the device ${ }^{(5,6)}$. Onlay bone is associated with a high risk of dehiscence during the healing process, with consequent exposure, contamination, and partial or total loss of the bone graft $^{7}$.

In 1976, Schettler $^{8}$ was the first who reported the sandwich osteotomy technique with interpositional bone grafting for vertical augmentation of the anterior edentulous mandible by maintaining the integrity of the lingual periosteum.

The rationale of any grafting procedure is to maximize the blood supply to the underlying bone graft and to prevent hypoxia with resulting ischemic changes at the distal portions of the flap which could eventually lead to surgical wound dehiscence ${ }^{9}$. The inlay grafting technique is a novel technique in which the inlayed graft would receive blood from 2 vascular pieces of bone, leading to faster new bone, with a low resorption level and high implant survival and success rates ${ }^{10}$.
A lack of comparative studies has made it difficult to choose the most reliable and predictable augmentation technique. Therefore, the aim of this study was to evaluate the validity of inlay grafting technique using bovine xenogenic graft versus autogenous graft.

\section{Purpose}

The aim of this study was to evaluate clinically, radiographically and histologically the use of interpositional xenogenic bone block graft in augmentation of atrophic posterior mandible by comparing it to the autogenous chin corticocancellous block for dental implant installation.

\section{MATERIALS AND METHODS}

Patient selection: twelve patients with atrophic posterior mandible (11 females and one male) were included in this study and their ages ranged from 27 to 53 years with a mean of 43.3 years. All patients had a minimum of $5 \mathrm{~mm}$ residual bone height above mandibular canal and adequate width of the alveolar ridge, they were divided randomly into two equal groups (6 patients each) according to the type of graft used for vertical augmentation, group I (control group) received autogenous chin bone block and group II (study group) received bovine bone block (Tutobone ${ }^{\circledR}$, Tutogen Medical Gmbh, Germany).

Patients were excluded from the study if met any of the following exclusion criteria (1) Systemic disorder that may compromise bone healing or osteointegration such as diabetes, radiotherapy, (2) Inter-arch space less than $12 \mathrm{~mm}$, (3) Presence of bone pathology, (4) Heavy smokers. All patients were evaluated and managed in Clinic of Oral and Maxillofacial Surgery Department, Faculty of Dentistry, Tanta University.

The purpose of this study was explained for all patients and informed consents were obtained according to the guidelines of human research 
adopted by the committee. Approval for this project was obtained from the Research Ethics Committee (REC) at Faculty of Dentistry, Tanta University.

Preoperative evaluation: The patients were evaluated for oral hygiene, mucosa (quality, color and presence of pathology) and occlusion. The height of residual alveolar bone extending from the mandibular canal to the alveolar crest was firstly measured by Cone beam computed tomography (CBCT).

\section{Augmentation procedure}

All patients were treated under local anesthesia, a paracrestal incision was made through the buccal mucosa respecting the emergence of the mental nerve. The full thickness flap was retracted without dissection toward the alveolar crest or the lingual side to preserve an adequate blood supply.

The horizontal osteotomy was made away from the alveolar crest leaving at least $2 \mathrm{~mm}$ above inferior alveolar canal using piezoelectric saw. Two oblique cuts were made in the osteotomized segment of the mandibular bone. The osteotomies were completed lingually with the use of bone osteotome. The segment was elevated preserving the lingual mucoperiosteum attachment.

According to the result of randomization, the autogenous corticocancellous chin block or the bovine bone block was interposed between the raised fragment and the mandibular basal bone after it was modeled to the desired shape and height, and the final height was measured intra-operatively. Titanium microplates and microscrews were used to fix the osteotomized crestal bone to the basal bone, maintaining the interposed graft fixed in its proper position until complete healing. The flap was sutured, after releasing incision was performed in periosteum.(Figure 1\&2)

Postoperative care: All patients in both groups were instructed for good oral hygiene measures using warm mouth wash 3 times a day. $1 \mathrm{~g}$ of Cefotaxime and Non-steroidal anti-inflammatory (Diclofenac sodium, Medical Union Pharmaceuticals, Ismailia, Egypt) were prescribed every 12 hours for 5 days. A soft diet was recommended for at least one month and removable prostheses was not allowed to be used.

\section{Follow up assessment}

Clinically: Patients were seen after one week for follow-up examination and sutures removal. They were recalled for additional postoperative follow ups weekly in the first month, and monthly in the subsequent 5 months for evaluation of wound healing, edema, infection, exposure of graft and paresthesia of lip.

Radiographically: Six months after augmentation, CBCT was taken to evaluate the final vertical height of the augmented site.

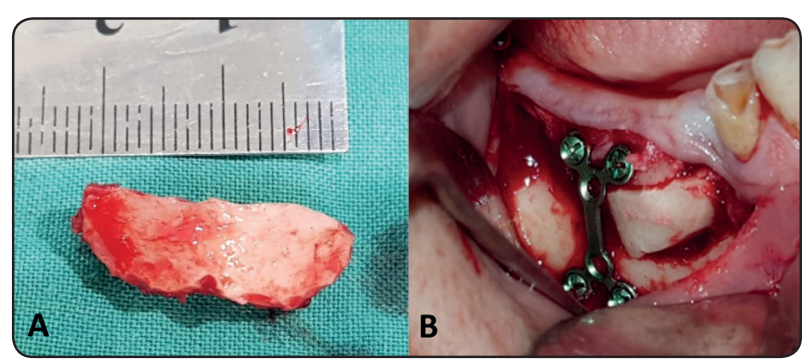

Fig. (1) Intraoperative photograph showing (a) the corticocancellous autogenous bone block was harvested from the chin, (b) the coronal osteotomized segment was elevated, autogenous graft was interposed and fixed with microplate.

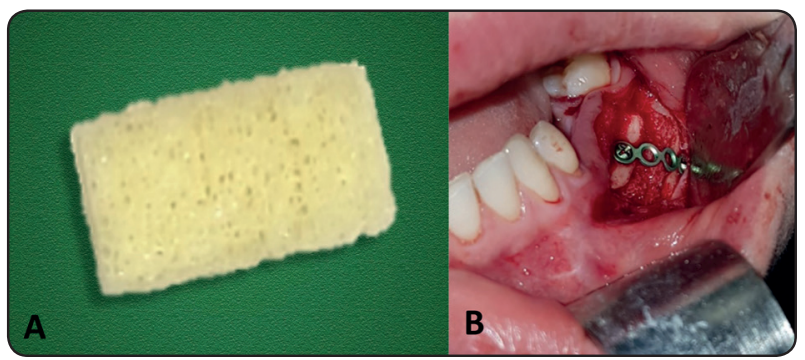

Fig. (2) Intraoperative photograph showing (a) bovine bone block after rehydration with saline (b) interpositional bovine bone block were fixed with titanium microplates and microscrews to both the basal bone and the transported segment. 
Histological evaluation: Six months after the augmentation procedure, the augmented site was exposed via crestal incision under LA. The screws and plate were removed, the bone core biopsies were retrieved by using trephine bur with internal diameter of $2 \mathrm{~mm}$ from the planned sites of dental implants, then stored immediately in $10 \%$ buffered formalin for histological evaluation. The specimens were decalcified in EDTA and after complete decalcification, they were infiltrated in paraffin wax blocks and they were cut into $5 \mu \mathrm{m}$ thick sections. The sections were stained by Hematoxylin and Eosin (H\&E) stain to be examined under light microscope.

Statistical analysis: All data was recorded, tabulated and statistically analyzed using computer software Statistical Package for social science (IBM Corp. Released 2013. IBM SPSS Statistics for windows, version 22. Armonk, NY: IPM Corp). Comparison between the two groups was performed using student t-test.

\section{RESULTS}

\section{Clinical results}

After augmentation procedure, wound healing was uneventful in all patients at the augmentation site except patient no. 1 group (I) who showed wound dehiscence with mild inflammation, which necessitates smoothening of the osteotomized coronal segment, local debridement and meticulous oral hygiene measures until soft tissue healing took place with secondary intension. All patients had transient paresthesia postoperative, but it resolved by the end of the first month which was most likely due to flap retraction.

\section{Radiographic results}

After six months of augmentation, the mean values of vertical bone height were $10.76 \pm 1.043 \mathrm{~mm}$ in group (I) and $11.24 \pm 0.3721 \mathrm{~mm}$ in group (II), which indicated significant increase in alveolar height in comparison to the preoperative mean values of height in group I $(5.783 \pm 0.392 \mathrm{~mm})$ and group II $(6.070 \pm 0.307 \mathrm{~mm})$.

The mean value of vertical bone height gain were $4.822 \pm 0.8286 \mathrm{~mm}$ and $5.320 \pm 0.2546 \mathrm{~mm}$ for group I and group II respectively. there was no statistical significant difference in height gain between both groups as $p>0.05$. (Table 1, Figure $3 \& 4)$

\section{Histological results}

After six months, histological evaluation of $\mathrm{H}$ $\&$ E stained sections revealed that the grafts in both

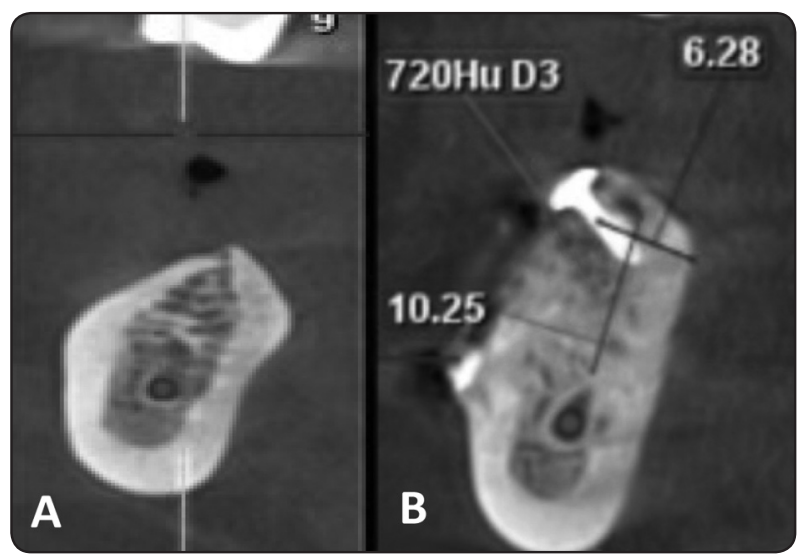

Fig. (3): a) Preoperative coronal CBCT showing deficient alveolar bone height, b) Six months postoperative CBCT showing increase the height of ridge and integration of the autogenous graft (group I)

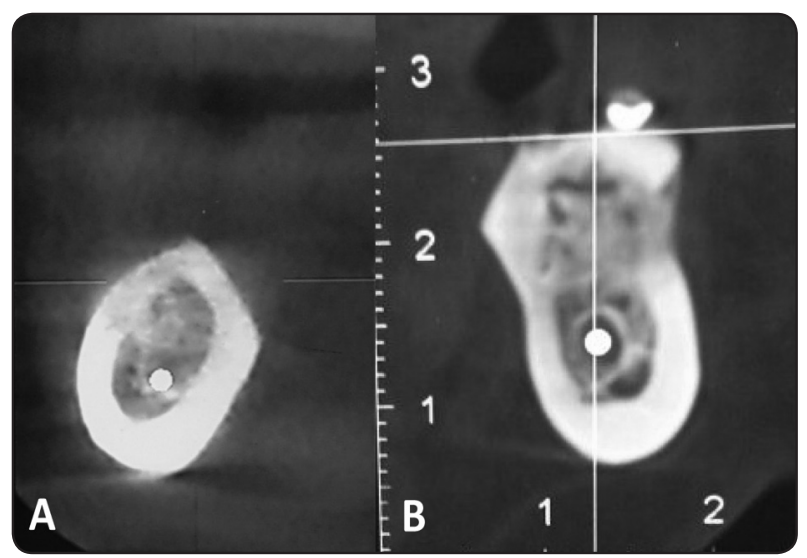

Fig. (4): a) Preoperative, b) Six months postoperative CBCT showing increase the height of ridge and integration of bovine bone block (group II). 
TABLE (1): Comparison of alveolar bone height between both groups at preoperative, six months postaugmentation intervals and the final height gain.

\begin{tabular}{|c|c|c|c|c|c|c|}
\hline & \multicolumn{4}{|c|}{ Alveolar bone height } & \multirow{2}{*}{\multicolumn{2}{|c|}{$\frac{\text { Bone height gain }}{\text { Six months post-augmentation }}$}} \\
\hline & \multicolumn{2}{|c|}{ Preoperative height } & \multicolumn{2}{|c|}{6 months post-augmentation height } & & \\
\hline & GI & GII & GI & GII & GI & GII \\
\hline $\mathrm{M} \pm \mathrm{SD}$ & $5.783 \pm 0.392$ & $6.070 \pm 0.307$ & $10.76 \pm 1.043$ & $11.24 \pm 0.3721$ & $4.822 \pm 0.8286$ & $5.320 \pm 0.2546$ \\
\hline $\mathrm{T}$ test & \multicolumn{2}{|c|}{0.8345} & \multicolumn{2}{|c|}{1.191} & \multicolumn{2}{|c|}{1.408} \\
\hline $\mathrm{P}$ - value & \multicolumn{2}{|c|}{$0.4235 \mathrm{~ns}$} & \multicolumn{2}{|c|}{$0.2612 \mathrm{~ns}$} & \multicolumn{2}{|c|}{$0.1894 \mathrm{~ns}$} \\
\hline
\end{tabular}

$p^{*}<0.05$ (significant)

groups showed a good level of osteointegration with an adequate extension of bonding surface between host bone and the graft particles.(Figure 5)

Autogenous graft (group I), there were a few numbers of autogenous graft remnants still present. Most area occupied by newly formed bone, these mature lamellar bones almost entirely filled the inter-particle space. High number of multinucleated giant cells (osteoclast cells) and resorption lacunae were present on the surface of newly formed bone

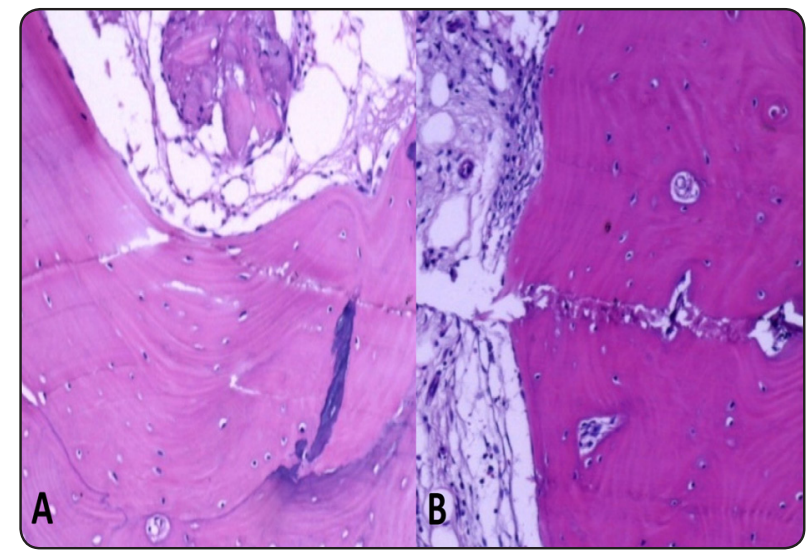

Fig. (5): Photomicrographs of a) autogenous specimen showing almost every particle of residual graft remnants surrounded by newly formed mineralized bone with osteoblasts lining it which indicate active bone formation and b) bovine specimen showing the remnants bridged by newly formed bone which consists of mature lamellar bone characterized by numerous osteocyte embedded within it and the bone marrow spaces were filled by fibro-cellular connective tissue (H\&E× 100). as well as the adjacent connective tissue which indicate active remodeling.

Bovine graft (group II), showed greater particles of graft remnants when compared with autogenous remnants and they were easily distinguishable from the other components of the regenerated tissue, but most of the bovine particles were united by newly formed bone which bridged the space between the graft particles. This bone consists of mature lamellar bone and woven bone which was characterized by numerous osteocyte embedded into the mineralized bone matrix. Numerous osteoblast cells were present all around the bone trabeculea indicating active new bone formation on the external surface.

\section{DISCUSSION}

Rehabilitation of partially or totally edentulous posterior mandible with implant-supported prosthesis has become a common practice in the last few decades, with reliable long-term results. However, local conditions of the posterior edentulous ridges may be unfavorable for implant placement due to insufficient bone height above the inferior alveolar nerve, which prevent dental implant placement without performing augmentation of alveolar bone height ${ }^{(11,12)}$.

This study was designed to determine if the xenogenic bovine bone graft can offer some similar results and successful outcomes in comparison to 
autogenous bone graft harvested from the mental symphysis in vertical augmentation of atrophic posterior mandible using the inlay technique.

The autogenous bone graft is considered the gold standard for bone augmentation techniques due to its properties of osteoconduction, osteoinduction, osteogenicity and osteointegration ${ }^{13}$. However, numerous disadvantages have also been described, such as donor site morbidity, increased operative time, soft-tissue injuries.

In order to eliminate complaints of donor site morbidity, we used the bovine bone graft as an alternative substitute to autogenous graft in this study. This was based on the previously documented properties by Rodriguez et al. ${ }^{14}$ and Martinez et al..$^{15}$ who found that bovine bone is up to $75 \%$ porous and has a high specific surface area of almost $100 \mathrm{~m} 2 / \mathrm{g}$ that results in increased angiogenesis, enhances new bone growth.

The inlay technique was selected for the augmentation rather than other methods based on the findings of the previous studies who reported that this technique provides well vascularized flaps to cover the grafted areas. The rationale of interposition of the bone graft between the osteotomized bony segments aimed to maximize the blood supply to the dependent bone graft ${ }^{(16,17)}$.

In this study, the minimum residual crest height ranged from 5 to $7 \mathrm{~mm}$ above the inferior alveolar nerve in all cases. According to the recommendations of Felice et al ${ }^{18}$ and Bormann et al. ${ }^{19}$ to avoid complications such as resorption or fracture of the transport segment or inferior alveolar nerve damage.

All osteotomies had been done using the piezosurgery device, according to Veecellotti ${ }^{20}$ who illustrated that the device has the ability to cut hard tissue without damaging soft tissues, providing a less-invasive surgical approach, as the location of mandibular canal in our patients was superficial because of the atrophy of the alveolar ridge which increase the risk of nerve injury if the surgical saws or burs were used

The grafts were fixed in our research with microplates as it is essential to provide mechanical support, minimize micromovements and the closer the adaptation of the graft to the recipient site, the better the integration of the graft with adequate bone formation and avoidance of fibrous tissue encapsulation. This matches with the recommendations of Uchida et al. ${ }^{21}$ and Tamimi et al..$^{22}$.

The recorded complication which happened in the current study was wound dehiscence which was treated with local irrigation, antibiotics and resolved after three weeks with resorption of part of the osteotomized cranial segment. This matched with other studies conducted by Marconcini et al. ${ }^{23}$, in which the most frequently documented complication is surgical wound dehiscence, exposure of the grafts and/or fixation appliances and grafted bone resorption.

Donor site morbidity in this study was tingling sensation in lower lip and anterior teeth even with taking all the precautions needed which was relieved by the end of the first postoperative month. This is in accordance with the finding of Raghoebar et al. ${ }^{24}$ and Pommer et al. ${ }^{25}$.

One of the advantages of using inlay technique in this study is the placement of the incision paracrestal so keeping the keratinized mucosa at the crest of ridge without interruption as a result the interface between implant shoulder and soft tissue is kept as similar as possible to the original. In consistent with the findings of the present study, lavive et al. ${ }^{26}$.

In the present study, the mean of vertical bone gain was $4.822 \pm 0.8286 \mathrm{~mm}$ (group I) and 5.320 $\pm 0.2546 \mathrm{~mm}$ (group II). this was in consistence with the results reported by Checchi et al. ${ }^{16}$ who registered a mean vertical bone gain of $5.5 \mathrm{~mm}$ and 
Marconini et al. ${ }^{23}$ who reported $5.9 \mathrm{~mm}$ vertical bone height gain 4 months after interpositional grafting procedure.

The limitations of height gain in inlay grafting was explained by Titsinides et al. ${ }^{27}$ who recommended keeping the soft tissue tension to minimal to avoid over stretching of the lingual periosteoum which may result in graft malperfusion and ischemic resorption of the graft and the native elevated bone segment and its dislodgement.

According to histological examination, the new bone formation was higher in autogenous graft when compared with bovine group, as it has greater osteogenic capacity than any other bone replacement material, and are therefore incorporated more easily without immunogenic responses. However, from a clinical point of view, it did not appear to provide any beneficial effect.

Coinciding with our findings, Jensen et al. ${ }^{(28,29)}$ have investigated that the autogenous bone provides a faster remodeling rate. So, it offers the possibility of a shorter healing time compared with the bovine bone mineral graft. But as a result of the minimal substitution rate of bovine bone mineral, it provides better stability of the graft height.

Coinciding with the results of the current study regarding to the successful augmentation using bovine graft when compared to autogenous graft, Felice et al., ${ }^{18}$ suggested that the bovine xenograft is a good alternative to the autogenous bone from the iliac crest as an interpositional graft material to vertically augment posterior atrophic mandibles using inlay technique.

Scarno et al., ${ }^{12}$ and Marconcini et al., ${ }^{23}$ also reported that the xenogenic bone block can be considered as a good material for bone regeneration in inlay grafting procedures in atrophic posterior mandibles, and allow implant placement in a second-stage surgery.

\section{CONCLUSION}

From the results of this study we can conclude that:

1. Inlay technique represent a successful procedure for augmentation of atrophic posterior mandible as it provides superior bone graft incorporation and less bone resorption, so a stable alveolar dimension, less soft tissue complications.

2. Both grafts achieved good results, however the bovine bone graft provides less invasive technique and avoids donor site morbidity.

\section{Funding}

The authors received no specific funding for this work.

\section{Conflicts of interest}

The authors declare no conflicts of interest.

\section{REFERENCE}

1. Tan WL, Wong TL, Wong MC, Lang NP. A systematic review of post-extractional alveolar hard and soft tissue dimensional changes in humans. Clin Oral Implants Res. 2012 Feb;23:1-21.

2. Luan Mavriqi: Sandwich Osteotomy of the Atrophic Posterior Mandible Prior to Implant Placement: A Case Report. Balkan J Dent Med, 58-63, 2014.

3. Jensen AT, Jensen SS, Worsaae N. Complications related to bone augmentation procedures of localized defects in the alveolar ridge. A retrospective clinical study. Oral Maxillofac Surg. 2016 Jun 1;20(2):115-22.

4. Jesus Torres, Faleh Tamimi, Mohammad Alkhraisat, Juan Carlos Prados-Frutos and Enrique Lopez-Cabarco. Bone Substitutes. In: Ilser Turkyilmaz, ed. Textbook of Implant Dentistry. The Most Promising Discipline of Dentistry. 1st ed. Europe: InTech, 2011, 91-107.

5. Zimmermann KA, Leblanc JM, Sheets K.T., Fox R. W., and Gatenholm P., "Biomimetic design of a bacterial cellulose/ hydroxyapatite nanocomposite for bone healing applications,"Materials Science and Engineering C, vol. 31, no. 1, pp.43-49, 2011. 
6. Herford, M. Lu, L. Akin, and M. Cicci u, "Evaluation of a porcine matrix with and without platelet-derived growth factor for bone graft coverage in pigs. Int $\mathrm{J}$ of Oral \& Maxillofac Implants, vol. 27, no. 6, pp. 1351-1358, 2012.

7. Pelo S, Boniello R, Moro A, Gasparini G, Amoroso PF. Augmentation of the atrophic edentulous mandible by a bilateral two-step osteotomy with autogenous bone graft to place osseointegrated dental implants. Int J Oral Maxillofac Surg. 2010 Mar 1;39(3):227-34

8. Schettler D. Sandwich-technique with cartilage transplant for raising the alveolar process in the lower jaw [in German].Fortschr Kiefer Gesichtschir 1976;20: 61-63.

9. Koymen R., Karacayli U., Gocmen-Mas N., ErtugrulKoymen C. Ortakoglu K., Gunaydin Y. and et al. Flap and incision design in implant surgery: clinical and anatomical study. Surg Radiol Anat. 31; 301-306, 2009.

10. Jensen OT, Kuhlke L, Bedard JF, White D. Alveolar segmental sandwich osteotomy for anterior maxillary vertical augmentation prior to implant placement. J Oral Maxillofac Surg. 2006 Feb 1;64(2):290-6.

11. Block MS, Haggerty CJ. Interpositional osteotomy for posterior mandible ridge augmentation. J Oral Maxillofac Surg. 2009 Nov 1;67(11):31-9.

12. Scarano A, Carinci F, Assenza B, Piattelli M, Murmura G, Piattelli A. Vertical ridge augmentation of atrophic posterior mandible using an inlay technique with a xenograft without miniscrews and miniplates: case series. Clin Oral Implants Res. 2011 Oct;22(10):1125-30

13. Nkenke E, Neukam FW. Autogenous bone harvesting and grafting in advanced jaw resorption: morbidity, resorption and implant survival. Eur J Oral Implantol. 2014 Jun 1;7(Suppl 2):S203-17.

14. Rodriguez A,Anastassov GE, Lee H, Buchbinder D, Wettan H. Maxillary sinus augmentation with deproteinated bovine bone and platelet rich plasma with simultaneous insertion of endosseous implants. J Oral Maxillofac Surg. 2003 Feb 1;61(2):157-63

15. Martinez A, Franco J, Saiz E, Guitian F. Maxillary sinus floor augmentation on humans: Packing simulations and 8 months histomorphometric comparative study of anorganic bone matrix and $\beta$-tricalcium phosphate particles as grafting materials. Mater Sci Eng: C. 2010 Jun 15;30(5):763-9.

16. Checchi V, Mazzoni A, Breschi L, Felice P. Reconstruction of an Atrophied Posterior Mandible with the Inlay Technique and Allograft Block Versus Allograft Particulate: A Case Report. Int J Periodontics Restorative Dent. 2015; 35:e20-7.

17. Yuce MO, Adali E, Turk G, Isik G, Gunbay T. Threedimensional bone grafting in dental implantology using autogenous bone ring transplant: Clinical outcomes of a one-stage technique. Nigerian journal of clinical practice. 2019 Jul 1;22(7):977

18. Felice P, Marchetti C, Iezzi G, Piattelli A, Worthington H, Pellegrino G, Esposito M. Vertical ridge augmentation of the atrophic posterior mandible with interpositional bloc grafts: bone from the iliac crest vs. bovine anorganic bone. Clinical and histological results up to one year after loading from a randomized-controlled clinical trial. Clin Oral Implants Res. 2009 Dec;20(12):1386-93

19. Bormann KH, Suarez-Cunqueiro MM, von See C, Tavassol F, Dissmann JP, Ruecker M, Kokemueller H, Gellrich NC. Forty sandwich osteotomies in atrophic mandibles: A retrospective study. J Oral Maxillofac Surg. 2011 Jun 1;69(6):1562-70.

20. Vercellotti T. Technological characteristics and clinical indications of piezoelectric bone surgery. Minerva Stomatol. 2004 May;53(5):207.

21. Schlee M, Steigmann M, Bratu E, Garg AK. Piezosurgery: basics and possibilities. Implant Dent. 2006 Dec $1 ; 15(4): 334-40$.

22. Uchida T, Yoshida T, Kashiwagi K, Lee S, Kobayashi W, Takahashi K, Murai M, Sato S, Ito K. Clinical, radiographic, and histologic evaluation of localized ridge augmentation using a mandibular bone block. Int $\mathrm{J}$ Periodontics Restorative Dent. 2008 Apr 1;28(2).

23. Marconcini S, Covani U, Giammarinaro E, Velasco-Ortega E, De Santis D, Alfonsi F, Barone A. Clinical success of dental implants placed in posterior mandible augmented with interpositional block graft: 3-year results from a prospective cohort clinical study. J Oral Maxillofac Surg. 2019 Feb 1;77(2):289-98.

24. Raghoebar GM, Louwerse C, Kalk WW, Vissink A. Morbidity of chin bone harvesting. Clin Oral Implants Res. 2001 Oct;12(5):503-7.

25. Pommer B, Tepper G, Gahleitner A, Zechner W, Watzek G. New safety margins for chin bone harvesting based on the course of the mandibular incisive canal in CT. Clin Oral Implants Res. 2008 Dec;19(12):1312-6. 
26. Laviv A, Jensen OT, Tarazi E, Casap N. Alveolar sandwich osteotomy in resorbed alveolar ridge for dental implants: a 4-year prospective study. J Oral Maxillofac Surg. 2014 Feb 1;72(2):292-303.

27. Titsinides S, Agrogiannis G, Karatzas T. Bone grafting materials in dentoalveolar reconstruction: A comprehensive review. Jpn Dent Sci Rev. 2019 Nov 1;55(1):26-32

28. Jensen T, Schou S, Svendsen PA, Forman JL, Gundersen HJG, Terheyden H, Holmstrup P. Volumetric changes of the graft after maxillary sinus floor augmentation with BioOss and autogenous bone in different ratios: a Sinus floor elevation and implants rdiographic study in minipigs. Clin Oral Implant Res. 2011 Nov 23: 902-910.

29. Jensen T, Schou S, Gundersen HJ, Forman JL, Terheyden H, Holmstrup P. Bone-to-implant contact after maxillary sinus floor augmentation with Bio-Oss and autogenous bone in different ratios in mini pigs. Clin Oral Implants Res. 2013 Jun;24(6):635-44. 